\title{
Headache in a young woman: do not forget Susac's syndrome
}

\author{
A. Kefi, F. Said, T. Ben Salem, M. Khedher, M. Khanfir, I. Ben Ghorbel, \\ M. Lamloum, M.H. Houman \\ Department of Internal Medicine Hospital of La Rabta, Faculty of Medicine of Tunis, \\ Tunis El Manar University, Tunis
}

\section{SUMMARY}

Susac syndrome is a rare disease characterized by the clinical triad of encephalopathy, branch retinal artery occlusion, and sensorineural hearing loss. This underdiagnosed condition needs to be considered in the differential diagnosis of a broad variety of disorders. An early diagnosis is important as treatment can halt disease progression and prevent permanent disability. Herein, we report a case of Susac syndrome in a 31-year-old woman and we highlight how challenging an early diagnosis was and the importance of an aggressive therapeutic approach, including the combination of steroids and other cytotoxic drugs.

Key words: Susac's syndrome; Headache; Young woman.

Reumatismo, 2017; 69 (3): 122-125

\section{INTRODUCTION}

usac's syndrome (SS), also known as retinocochleocerebral vasculopathy, is a rare condition that was first reported in 1973 (1). Although SS pathophysiology remains mysterious, it is now thought that it is most probably an immune-mediated endotheliopathy affecting the precapillary arterioles of the brain, retina, and inner ear (2). Although the clinical triad of encephalopathy, branch retinal artery occlusions (BRAO), and sensorineural hearing loss, representing the main feature of $\mathrm{SS}$, is increasingly recognized, SS remains often underdiagnosed particularly at the early stage (3). Herein, we report a case of a 30-year-old woman with SS and we highlight the difficulties of an early diagnosis and adequate treatment.

\section{CASE REPORT}

A 31-year-old woman was admitted to our department with a history of two months of severe headache, blurry vision and sudden painless vision deterioration in the left eye (LE). A review of the remaining medical history was unremarkable. Particularly, there were no hearing difficul- ties, no cognitive impairment, no memory loss, no vertigo, no psychiatric disorders and no weakness. The patient denied any infectious exposure or seizure. On physical examination, she was afebrile, and conscious. Blood pressure was normal and all peripheral pulses were palpable and symmetrical. There were no pertinent findings especially from examination of cardiovascular, pulmonary and neurological systems. Ophthalmic examination in the LE revealed BRAO with retinal cloudy swelling at the upper part of the macula. The rest of the ophthalmic examination showed no abnormalities. The right eye (RE) was completely normal with vision of 10/10. Laboratory tests showed no relevant alterations in blood cell count, hemostasis tests, renal and liver function, electrolytes, or lipid panel. There was no biological inflammatory syndrome. Tests for antinuclear antibodies (ANA), anti double stranded DNA (dsDNA), extractable nuclear antigen (ENA) panel, anti-neutrophil cytoplasmic antibodies (ANCA), anti-prothrombin antibodies and antiphospholipid antibodies were negative.

Electrocardiogram, and echography of the supra-aortic trunks showed no ab- 
normalities. Brain MRI with intravenous contrast revealed three small hyperintense foci on $\mathrm{T} 2$ weighted images and contrast enhancement involving the anterior third of the corpus collosum suggesting micro infracts. There were multiple hyperintense lesions in the right posterior cortical white matter, without any restricted diffusion. Fluid-attenuated inversion recovery (FLAIR) showed punctiform images located in the periventricular white matter and the semi-oval centers. Cerebrospinal fluid was normal. Considering all these clinical and investigational findings in a young woman without any cardiovascular risk factors, and after ruling out embolic cardiopathies, vasculitis, connective tissue disease, infections and hypercoagulable state, Susac syndrome was the most likely diagnosis.

Despite the absence of hearing impairment complaints, the audiometry study revealed hearing loss on the right side at low frequency perception with a loss of $30 \mathrm{~dB}$. The diagnosis of Susac syndrome was made. Our patient was started on a pulse of methylprednisolone $(1000 \mathrm{mg} / \mathrm{d}$ for 3 days), followed by an oral dose of $1 \mathrm{mg} / \mathrm{kg}$ daily, which was slowly tapered. The clinical outcome was favorable. In fact, we noticed the disappearance of headaches and the improvement of visual activity.

One year later, the patient returned with a relapse of disease including headache and a new BRAO in the right eye with retinal oedema and peripheral vasculitis. We decided to start a treatment consisting of methylprednisolone $1000 \mathrm{mg}$ per day for 3 consecutive days, followed by oral prednisolone: $1 \mathrm{mg} / \mathrm{kg}$ daily; in conjunction with cyclophosphamide (6 monthly infusions of $750 \mathrm{mg} / \mathrm{m}^{2}$ ). After 4 weeks, we started a very slow tapering.

The patient responded very well to the treatment with full recovery from her symptoms. Six months later, a brain MRI showed significant resolution of corpus collosum lesions and disappearance of white matter lesions. Mycophenolate mofetil was then started as maintenance therapy with good evolution.

\section{DISCUSSION}

Susac syndrome is an orphan disease that is characterized by the clinical triad of encephalopathy, BRAO and hearing loss (3). SS was first described by John O. Susac in 1979 in two young women with the classic triad, but was designated as Susac syndrome by Hoyt in 1986 (1). The age of our patient was characteristic for the development of initial symptoms during the natural course of SS. In fact, this endotheliopathy affects mainly middle-aged women (20-40 years old) (3).

In our case, SS was manifested as a full characteristic clinical triad on presentation, even if headache and vision loss were the most important symptoms. Unlike our case, the clinical triad occurs at disease onset in only a minority of patients. In fact, the largest meta-analysis published by Dorr et al. found that only $13 \%$ of patients show the clinical triad at disease onset (4). That is why SS may be underdiagnosed. To help physicians to diagnose this syndrome early in its course, Vishnevskia-Dai et al. proposed a clinical classification of SS, based on literature data (case reports, reviews and meta-analysis) (5).

The pathogenesis of SS remains unclear but immune mechanisms, vasospastic phenomena and coagulopathy have all been implicated. Now, SS is considered as an autoimmune microangiopathy, with an underlying pathology identified as a non-inflammatory microangiopathy causing small infarcts in the brain, cochlea and retina. Also interesting was the hypothesis that SS represents an autoimmune endotheliopathy/ coagulopathy similar to the catastrophic antiphospholipid syndrome. It is suggested that previous infections acting as a trigger may play a part in the pathogenesis of SS (6). Some authors have hypothesized that anti-endothelial cell antibodies could play a role in either mediating or reflecting the endothelial cell injury in Susac's syndrome because they have been reported in Susac's syndrome patients. However, until now, no evidence has been established $(2,7,8)$.

As for clinical manifestations of SS, headache, severe and often migrainous, is the 
most common prodromal symptom and may appear several months before the development of other symptoms including cognitive changes, memory loss, confusion, ataxia, dysarthria, vertigo, corticospinal tract dysfunction, seizures, pyramidal signs, weakness, hyperreflexia and psychiatric disorders (personality change and bizarre behavior) (6). Ophthalmological involvement includes many types of disturbances such as photopsia, black spots, scintillating scotomas and even severe visual loss, which was reported in our patient (9). These symptoms are often caused by BRAO (6). Hearing loss is the major otological manifestation. It may be unilateral or bilateral, and is usually asymmetrical involving mainly the low and mid-frequencies. Hearing loss can be a dramatic and severely disabilitating feature of SS as it is often irreversible and may require cochlear implants or lifelong hearing devices (6). Other manifestations include myalgia, arthralgia and skin lesions (10). A broad spectrum of differential diagnosis includes autoimmune diseases (systemic lupus erythematosus, neuro-Behçet disease) demyelinating diseases (multiple sclerosis, acute disseminated encephalomyelitis), and vascular occlusion (atherosclerosis or embolic disorders). Even if MRI findings may mimic other neurological disorders, the observance, as in our case, of lesions of the corpus callosum, which is always involved in SS, enhances the suspicion of SS, especially when there are coexisting auditory or BRAO problems (11).

It seems important to emphasize that, given the scarcity of the clinical triad at SS onset, a diagnosis based solely on the presence of the complete triad may not be appropriate. That is why a targeted search for absent components of the triad (throughout retinal fluorescein angiography and audiometry) in patients showing encephalopathy of unknown origin is essential.

Because of the rarity of SS and the diagnosis delay, all our knowledge to date is based on clinical experience, case reports, and small case series. Consequently, the best treatment strategy still needs to be defined (controlled clinical trials).
In our case, despite corticosteroid therapy, the disease relapsed so that immunosuppressive treatment was needed. Our experience with this patient and literature data demonstrate that SS has to be treated early, aggressively and for a sufficiently long time to prevent further damage and relapses (2). Treatment should include immunosuppression with steroids and cytotoxic drugs such as cyclophosphamide, azathioprine, mycophenolate mofetil and methotrexate. Some patients with severe cases may need intravenous immunoglobulins. Antithrombotic measures and antiplatelet agents should be strongly considered $(12,13)$. Although there is agreement that high-dose corticosteroids should be the first line therapy and early, aggressive, sustained immunosuppressive treatment may markedly improve outcomes, it remains unclear as to how much immunosuppressive medication is required and for how long. As described in our case, some authors have reported the use of mycophenolate mofetil with a good evolution $(12,14,15)$. As for corticosteroid therapy, we would use it in every patient starting with a pulse of highly dosed methylprednisolone (1000 mg/d for 5 days), then an oral dose of $1 \mathrm{mg} / \mathrm{kg}$ daily, followed by a slow tapering, depending on the type and course of the patient's disease. We recommend not tapering too fast. To summarize, the most important step for correct treatment seems to be correct and early diagnosis (12). Careful control and follow-up of the patient is strongly recommended to recognize and treat relapses as early as possible $(6,12)$.

\section{CONCLUSIONS}

$\mathrm{SS}$ is a challenging disorder to recognize initially. Although SS is characterized by the clinical triad, it is probably underdiagnosed due to the clinical presentation, as patients often present only part of this triad. The range of physicians who should be aware of SS and be capable of making the correct diagnosis as soon as possible is wide. This case report highlights the need for an aggressive therapeutic approach to prevent further damage and relapses. In ad- 
dition, this case supports recommendation of working together via an interdisciplinary approach in the follow-up controls and the treatment of the patient.

Conflict of interest: nothing to declare.

\section{REFERENCES}

1. Susac JO, Egan RA, Rennebohm RM, et al. Susac's syndrome: 1975-2005 microangiopathy/autoimmune endotheliopathy. J Neurol Sci. 2007; 257: 270-2.

2. Kleffner I, Duning T, Lohmann H, et al. A brief review of Susac syndrome. J Neurol Sci. 2012; 322: 35-40.

3. Grygiel-Górni AKB, Puszczewicz M, Czaalicka E. Susac syndrome-clinical insight and strategies of therapy. Eur Rev Med Pharmacol Sci. 2015; 19: 1729-35.

4. Dorr J, Krautwald S, Wildemann B, et al. Characteristics of Susac syndrome: a review of all reported cases. Nat Rev Neurol. 2013; 9: 307-16.

5. Vishnevskia-Dai V, Chapman J, Sheinfeld R, et al. Susac syndrome: clinical characteristics, clinical classification, and long-term prognosis. Medicine (Baltimore). 2016; 95: e522.

6. García-Carrasco M, Mendoza-Pinto C, Cervera R. Diagnosis and classification of Susac syndrome. Autoimmun Rev. 2014; 13: 347-50.

7. Magro CM, Poe JC, Lubow M, Susac JO.
Susac syndrome: an organ-specific autoimmune endotheliopathy syndrome associated with anti-endothelial cell antibodies. Am J Clin Pathol. 2011; 136: 903-12.

8. Jarius S, Neumayer B, Wandinger KP, et al. Antiendothelial serum antibodies in a patient with Susac's syndrome. J Neurol Sci. 2009; 285: 259-61.

9. Egan RA, Hills WL, Susac JO. Gass plaques and fluorescein leakage in Susac syndrome. J Neurol Sci. 2010; 299: 97-100.

10. Gertner E, Rosenbloom MH. Susac syndrome with prominent dermatological findings and a prompt response to intravenous immunoglobulin, steroids, and rituximab: a case report. J Med Case Rep. 2016; 10: 137.

11. Greco A, De Virgilio A, Gallo A, et al. Susac's syndrome--pathogenesis, clinical variants and treatment approaches. Autoimmun Rev. 2014; 13: 814-21.

12. Klein M, Illies T, Georgi S, et al. Aggressive immunotherapy in Susac's syndrome. Nervenarzt. 2009; 80: 1502-5.

13. García-Carrasco M, Jiménez-Hernández C, Jiménez-Hernández M, et al. Susac's syndrome: an update. Autoimmun Rev. 2011; 10: 548-52.

14. Bitra RK, Eggenberger E. Review of Susac syndrome. Curr Opin Ophthalmol. 2011; 22: 472-6.

15. Entezari M, Karimi S, Feizi M. Progressive Susac syndrome with bilateral visual loss and disability. Indian J Ophtalmol. 2016; 64: 678-80. 